\title{
LA PARTICIPATION DES ENFANTS ET DES JEUNES DANS LES ÉCOLES ESPAGNOLES : EXPÉRIENCES À SÉVILLE
}

Francisco F. García Pérez, Nicolás de Alba Fernández, Pilar Estada Aceña, Trinidad Herrero Campos

Armand Colin | « Carrefours de l'éducation »

2009/2 n²8 | pages 111 à 122

ISSN 1262-3490

Article disponible en ligne à l'adresse :

http://www.cairn.info/revue-carrefours-de-l-education-2009-2-page-111.htm

\section{Pour citer cet article :}

Francisco F. García Pérez et al., « La participation des enfants et des jeunes dans les écoles espagnoles : expériences à Séville », Carrefours de l'éducation 2009/2 ( ${ }^{\circ}$ 28), p. 111-122.

DOI 10.3917/cdle.028.0111

Distribution électronique Cairn.info pour Armand Colin.

(C) Armand Colin. Tous droits réservés pour tous pays.

La reproduction ou représentation de cet article, notamment par photocopie, n'est autorisée que dans les limites des conditions générales d'utilisation du site ou, le cas échéant, des conditions générales de la licence souscrite par votre établissement. Toute autre reproduction ou représentation, en tout ou partie, sous quelque forme et de quelque manière que ce soit, est interdite sauf accord préalable et écrit de l'éditeur, en dehors des cas prévus par la législation en vigueur en France. Il est précisé que son stockage dans une base de données est également interdit. 


\title{
La participation des enfants et des jeunes dans les écoles espagnoles: expériences à Séville
}

\author{
$\Delta$ Francisco F. García Pérez, Nicolás de Alba Fernández, \\ Pilar Estada Aceña, Trinidad Herrero Campos \\ ffgarcia@us.es-ndealba@us.es \\ pilarestada@educa.org-trinidadherrero@educa.org
}

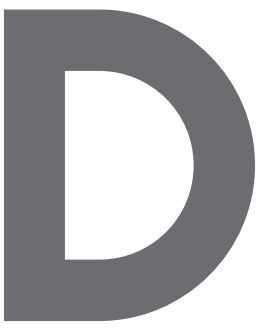

ans le système scolaire espagnol ${ }^{1}$, l'éducation civique a été fortement marquée par la dictature franquiste qui a promulgué l'endoctrinement politique incarné, à l'école, par une matière scolaire intitulée «Formation de l'esprit national ». Ainsi, l'éducation civique a acquis une connotation de propagande idéologique qui persiste encore dans quelques secteurs sociaux ${ }^{2}$. Cette situation a généré un blocage de la population vis-à-vis de ce type d'éducation et a rendu difficile son développement du point de vue pédagogique et didactique. En dépit de diverses tentatives législatives réalisées les dernières décennies, les contenus à caractère civique ne sont stabilisés ni comme aire disciplinaire ni comme axe transversal ${ }^{3}$. Ces contenus ont commencé à avoir une présence lors de l'approbation de la Constitution de 1978 et, surtout, lors de l'implantation en 1990 de la loi organique générale du

1. Ce travail est le résultat partiel du Projet R + D, avec référence SEJ2006-08714/EDUC, financé par le ministère de l'Éducation et de la Science d'Espagne (MEC) et par les fonds FEDER, intitulé «Éducation à la citoyenneté et formation du professorat: Difficultés et possibilités d'éduquer dans la participation des citoyens » ("Educación para la ciudadanía y formación del profesorado: Dificultades y posibilidades para educar en la participación ciudadana »). Texte traduit en français par Margarita Peña Rodríguez. Révision: Maria Pagoni et Philippe Haeberli.

2. Comme c'est le cas de la plus grande partie de la hiérarchie catholique ainsi que des groupes sociaux conservateurs, qui voient actuellement l'éducation à la citoyenneté comme « une propagande du gouvernement socialiste .

3. Pour une vision générale de l'éducation à la citoyenneté en Espagne on peut consulter, par exemple, Naval, Print and Iriarte, 2003, Martín, 2006, Jiménez et González, 2007, Bolívar, 2005. Cette vision peut être mise en rapport avec la situation européenne dans des publications comme celle d'Eurydice, 2005. 
système éducatif (LOGSE), qui a donné un rôle éducatif important aux matières transversales.

\section{Particularités du contexte espagnol: histoire, difficultés et influences}

Actuellement, la loi organique d'éducation (LOE) tente d'incorporer une matière intitulée «Éducation à la citoyenneté » dans le système scolaire ${ }^{4}$. Celle-ci répond sans doute au besoin d'intégrer dans le curriculum scolaire ce type de contenus. Les difficultés sont toutefois nombreuses en raison du contexte mentionné plus haut ainsi que, plus généralement, de la forme scolaire qui rend la formation des enseignants inadéquate pour l'enseignement des savoirs et compétences spécifiques à ce domaine.

L'inexistence d'une matière d'éducation civique dans le curriculum ne signifie pas que n'existent pas ou n'ont pas existé, de multiples expériences et projets concernant l'éducation des élèves à la citoyenneté et, plus concrètement, en rapport avec la participation. Cependant, ces expériences se sont développées surtout en tant qu'activités complémentaires aux activités scolaires ou dans le cadre de programmes éducatifs à caractère non formel, promus par des associations diverses, par des institutions municipales, etc. Les expériences espagnoles de participation s'intègrent dans différents types de projets et d'expériences qui se sont développés ou qui sont en cours de réalisation dans d'autres pays européens. Nous pouvons identifier au moins trois projets de ce type:

Le mouvement des «Villes éducatrices ${ }^{5}$ ». Ce réseau de villes, implanté dans plusieurs pays du monde et avec une forte expansion en Espagne, travaille autour de l'idée que la ville, comme d'autres espaces sociaux, est un espace potentiellement éducatif, et que, par conséquent, cette potentialité doit être exploitée et dévelop-

4. La LOE (BOE, 2006) se trouve en phase de mise en œuvre par les Communautés autonomes de l'État espagnol. La LOE prévoit au troisième cycle de l'éducation primaire (élèves de 10 à 12 ans) une matière d'éducation à la citoyenneté et aux droits de l'Homme; à l'éducation secondaire obligatoire (élèves de 12 à 16 ans) l'éducation à la citoyenneté se distingue en deux matières: l'une d'entre elles a le même nom qu'en primaire et est enseignée dans une des trois premières classes du secondaire; l'autre matière a le nom d'éducation éthique-civique, et est enseignée à la quatrième classe de ce niveau d'enseignement. Le nombre d'heures de ces matières est, en tout cas, très faible: 50 heures annuelles pour l'éducation primaire; et, dans le secondaire, 35 heures annuelles pour l'éducation à la citoyenneté et aux droits de l'Homme et 35 heures annuelles pour l'éducation éthique-civique. Au baccalauréat (entre 16 à 18 ans) l'éducation à la citoyenneté fait partie de la philosophie au sein d'une matière avec le nom philosophie et citoyenneté. Pour plus d'informations à ce propos voir: http://www.politischebildung.ch/schweiz-international/international/espagne/ 5. Le mouvement de «villes éducatrices » (http://www.edcities.bcn.es) naît comme tel dans le premier congrès international, tenu à Barcelone, en 1990, proposé par la mairie. 70 villes et 21 pays y ont participé, ont signé la "Charte de villes éducatrices » et se sont engagés à accomplir leurs principes. Cette charte a été révisée et actualisée en 1994. Le congrès de Göteborg (1992) a donné lieu à la constitution de l'« Association internationale de villes éducatrices». Tous les deux ans, des congrès internationaux (le IX a eu lieu à Lyon en septembre 2006) sont organisés. 
pée par les gouvernements municipaux et par la société en général. Il s'agit donc d'intégrer aux fonctions traditionnelles du gouvernement municipal (économique, sociale, politique et de prestation de services) une fonction éducative, dont l'objectif serait la formation, la promotion et le développement de tous ses habitants, et en priorité des enfants et des jeunes.

Le projet " La città dei bambini ${ }^{6}$ " (" La ville des enfants »), dans sa philosophie générale, défend une nouvelle façon de vivre la ville et de rendre celle-ci « habitable » pour les enfants, et, par conséquent, pour tous les habitants. Concrètement, ce projet promeut la participation directe des enfants dans la gestion des affaires de leur ville, de manière que leur voix soit entendue afin de pouvoir construire une ville meilleure (Tonucci, 1996). Le projet prend racine dans la féconde tradition pédagogique italienne ("Movimento di Cooperazione Educativa », expériences pédagogiques dans les écoles de la région d'Emilia-Romagna, etc.), qui a également eu une grande influence sur l'Espagne.

D'autres projets et d'autres expériences constituent des références pour le contexte espagnol en ce qui concerne la participation, comme les expériences développées en France et en Suisse ${ }^{7}$. Dans celles-ci, la structure participative reproduit d'un point de vue formel le modèle adulte de participation en permettant aux enfants d'être consultés à propos des projets qui les concernent et de jeter les bases d'un certain apprentissage actif de la démocratie. Ces dernières années, l'influence du modèle adulte, des « Budgets Participatifs », inspiré principalement des pratiques brésiliennes (comme celle de Portoalegre ou de São Paulo) et mis en œuvre dans de nombreuses villes espagnoles - comme c'est le cas de Séville elle-même -, a suscité un certain élan vers une participation citoyenne, ce qui a produit, pareillement, l'augmentation des expériences éducatives de ce type avec des enfants et des jeunes.

\section{Un cadre de référence pour la participation}

La participation constitue un axe central de l'éducation à la citoyenneté. De là l'intérêt éducatif de la recherche sur la participation des enfants et des jeunes comme citoyens, ainsi que sur le rôle des enseignants dans l'éducation à la participation. C'est actuellement le principal objet de recherche de notre équipe. À ce sujet, nous présenterons quelques réflexions sur des pratiques mises en place à Séville.

Notre recherche s'appuie sur une conception de la citoyenneté de caractère « globale », qui se concrétise, fondamentalement, par trois aspects: remettre en

6. Ce projet (http://www.lacittadeibambini.org/), promu par le pédagogue italien Francesco Tonucci, a commencé à se développer à Fano, la ville natale de Tonucci, et a été étendu à d'autres communes de l'Italie et de l'Espagne, et à d'autres pays de l'Europe et de l'Amérique Latine (spécialement l'Argentine et le Brésil).

7. Cependant les conseils d'élèves conçus comme lieu de participation scolaire n'ont pas été développés dans le système scolaire espagnol. 
question les limites des patries, les limites des domaines éducatifs conventionnels et les limites entre éducation formelle et non formelle.

En effet, l'éducation contribuerait à la construction d'une citoyenneté conçue comme mondiale, puisque les réalités de notre monde (globalisation, médiatisation, graves problèmes environnementaux...) exigent des citoyens une réponse à l'échelle planétaire, qui puisse dépasser les étroites frontières de chaque pays, comme cela est réclamé par diverses instances ${ }^{8}$. Toutefois, la revendication d'une échelle planétaire ne peut pas négliger complètement l'échelle locale, à laquelle doit inévitablement se situer l'intervention des élèves.

L'idée de citoyenneté dépasserait, pareillement, les limites traditionnelles des champs du savoir impliqués dans l'enseignement, en incorporant toute une série de dimensions ou de facettes qui permettraient d'articuler une approche éducative plus complexe. En ce sens, il semble indispensable de compter sur une dimension d'information politique, sociale et institutionnelle, autant que sur une perspective historique et actuelle ${ }^{9}$. Mais l'éducation à la citoyenneté requiert une dimension plus liée à l'action, qui pourrait être formulée comme éducation à la participation citoyenne, comprise dans un vaste sens et à différentes échelles (dans le quartier lui-même, dans la ville, dans l'État, dans le monde...). Cette dimension inclurait, parmi d'autres aspects, l'éducation politique et, logiquement, l'éducation à la démocratie, ainsi que la perspective éthique.

Cette approche ne suppose pas de se passer de la contribution de base de certaines disciplines scolaires - comme c'est le cas, spécialement, de l'histoire mais de profiter des savoirs que ces disciplines apportent, surtout quant à leur potentialité pour le traitement de problèmes sociaux significatifs. On travaillerait, par conséquent, les disciplines non comme une fin en soi mais, plutôt, comme un outil privilégié pour la compréhension des problèmes du monde. Cette perspective faciliterait la connexion avec d'autres domaines de connaissance et permettrait un meilleur transfert des connaissances à des contextes non scolaires.

Dans cette mesure l'approche globale que nous réclamons requiert, aussi, le concours d'autres dimensions significatives: celle relative à la coexistence et celle relative à l'engagement dans les problèmes sociaux et environnementaux nous semblent, au minimum, indispensables. On pourrait dégager, ainsi, pour cette approche d'éducation à la citoyenneté, une éducation à la coexistence, une éducation à la paix, et, en ce qui concerne le compromis avec le problème environnemental, serait également requise une éducation au développement durable, en incorporant, depuis une nouvelle optique, des aspects de la traditionnelle éducation au développement et des nouvelles perspectives de l'éducation

8. Cette idée a été largement développée par des auteurs comme E. Morin, dans ses différentes œuvres, dont le rapport pour l'Unesco (Morin, 1999). On peut voir également, à ce sujet, García, 2005 .

9. Cette perspective pourrait avoir un lien plus grand avec des contenus des sciences juridiques, qui n'ont pas suscité un grand intérêt dans le contexte espagnol, contrairement au contexte français (voir, par exemple, Audigier et Lagelée, 1996 et Robert, 1999). 
pour l'environnement. Dans ces dimensions seraient inclus, par conséquent, la sensibilité et le sens critique face aux inégalités, ainsi que l'engagement pour un monde plus juste.

Finalement, il est évident qu'une éducation à la citoyenneté de ce type, ne peut pas être réduite au domaine de l'éducation formelle et, moins encore, à l'espace curriculaire étroit d'une matière avec une faible présence scolaire, comme c'est le cas en Espagne. En effet, il nous semble indispensable que certaines propositions développées dans le domaine de l'éducation non formelle, soient prises en considération par l'école pour travailler le contenu de la participation ou d'autres contenus en rapport avec le monde des valeurs ${ }^{10}$. Ce type d'expériences présente quelques avantages par rapport à certaines caractéristiques de l'école traditionnelle: une organisation flexible des espaces et des temps; une conception des contenus plus ouverte et liée aux problèmes sociaux proches; une conception des activités plus variée; un rôle plus actif des apprentis et du formateur lui-même... En ce sens, et en étant conscients qu'on ne peut pas transférer facilement au contexte scolaire des expériences produites en dehors de celui-ci, il serait nécessaire de tenir compte de ces possibles contributions.

Comme nous l'avons exposé, la dimension participative est fondamentale pour l'éducation à la citoyenneté dans notre monde. Cependant, l'intégration de la participation aux processus éducatifs présente quelques difficultés, comme, par exemple, le fait que l'activité participative peut entrer en conflit avec la forme scolaire ${ }^{11}$, et, concrètement, avec le monde des savoirs académiques qui se base plus sur l'acquisition et le contrôle des connaissances que sur l'activité effective des élèves.

La participation, telle que nous la concevons, doit surtout être liée à l'engagement et, par conséquent, à l'action sociale, dans des domaines où les élèves ont des possibilités d'intervenir ${ }^{12}$. De plus, un des objectifs de l'éducation à la participation serait d'élargir ces domaines pour les citoyens-élèves. Le défi serait de lier la participation non seulement à l'action concrète mais au monde des valeurs et des attitudes, en consolidant de cette façon l'apprentissage de la citoyenneté comme une construction complexe et en garantissant le transfert de ce que l'on a appris à l'école au contexte citoyen réel.

10. Pour une analyse beaucoup plus détaillée de cette perspective de perméabilité entre les domaines de l'éducation formelle et non formelle, en rapport avec le champ de l'éducation au développement, on peut consulter De Alba, 2005.

11. Divers auteurs ont attiré l'attention sur les contraintes de « la forme scolaire » (voir, par exemple dans Éduscol, 2006).

12. Le besoin d'établir un lien entre les activités scolaires et les activités sociales comme citoyens, en améliorant le climat des salles de classe et en faisant de l'école un lieu de pratique de la démocratie, a été souligné par beaucoup d'auteurs (voir, par exemple, Hahn, 2001, Dam and Volman, 2004, Torney-Purta and Barber, 2005, Delval, 2006). 


\section{Quelques expériences pour intégrer la participation au contexte scolaire}

Nous allons exposer deux expériences qui se déroulent en Espagne, dans la province de Séville (dans la capitale et dans quelques villes de la province) et qui, nous croyons, peuvent révéler les possibilités et les difficultés des projets qui essaient de mettre en place l'éducation des élèves à la participation. Il s'agit $\mathrm{du}$ «Parlement des Jeunes» («Parlamento Joven ») et du projet des « Budgets Participatifs de Séville: Laboraforo» («Presupuestos Participativos de Sevilla: Laboraforo »). Ces expériences combinent une dimension plus spécifiquement pédagogique, qui consiste à éduquer aux valeurs démocratiques, avec une autre plutôt politique, qui consiste à créer un espace public de participation avec les enfants, les adolescents et les jeunes dans leur ville. Tous les deux se centrent sur la participation dans le domaine municipal et ont une dynamique similaire, bien que l'expérience de Laboraforo a la spécificité de travailler la participation des enfants, des adolescents et des jeunes par rapport aux budgets municipaux, dans le cadre de l'initiative des « Budgets participatifs » de l'aire de participation citoyenne de la mairie de Séville.

Les conclusions, à caractère partiel, que nous recueillions ici, ainsi que les réflexions que nous réalisons à partir de celles-ci, procèdent, surtout, de la recherche sur l'expérience du «Parlement des Jeunes». Ces conclusions ont été mises en rapport avec les conclusions dérivées du projet des « Budgets Participatifs de Séville: Laboraforo », développée par d'autres chercheurs de l'Université de Séville. Dans le cas du « Parlement des Jeunes » nous utilisons une méthodologie qui intègre des dimensions quantitatives et qualitatives, grâce à l'emploi des questionnaires répondus par professeurs des établissements éducatifs impliqués et par techniciens responsables du projet éducatif, l'observation des activités des élèves et, surtout, la réalisation des sessions de groupes de débat avec des professeurs. Tandis que les questionnaires ont été passés à un échantillon de population de l'ensemble de villes impliquées dans le programme, les observations d'activités des élèves et les sessions de groupes de débat se sont centrées en quatre établissements situés en trois des villes où le programme se développe. Les contenus qui sont objet de recherche ont rapport, principalement, aux aspects et catégories suivantes: le rôle des élèves en ce qui concerne la participation; l'intégration de la participation dans le curriculum scolaire; les difficultés des professeurs dans le développement des projets éducatifs d'éducation à la participation.

Le Parlement des jeunes ${ }^{13}$ est un programme (Ferreras et al., 2006) qui concerne les élèves de l'Education Secondaire Obligatoire (entre 12 et 16 ans) et prétend créer une structure stable de participation dans plusieurs villes de la province de Séville - actuellement vingt-trois, dont six sont dans leur première année, six

13. Proposé par le collectif «Argos, Projets éducatifs », ce projet a été assumé comme programme par l'aire de jeunesse, innovation et formation pour l'emploi de la députation provinciale de Séville. 
dans la deuxième et onze dans la troisième. Il s'agit, plus précisément, d'intégrer la perspective des jeunes aux politiques locales et de créer un espace où les adolescents et les jeunes puissent mettre en commun la vision qu'ils ont de leur ville, poser les problèmes qui les affectent et faire des propositions d'amélioration pour les résoudre.

En général, le système d'élection des jeunes parlementaires se réalise selon certains critères. Le Parlement des Jeunes de chaque municipalité est normalement composé par vingt jeunes. Ce groupe de jeunes doit être composé par un nombre égal de garçons et de filles et représenter un grand nombre de quartiers, secteurs, ambiants et collectifs sociaux de la municipalité. Pour cela, on recourt au tirage au sort combiné au choix direct de quelques membres de la part des éducateurs responsables pour cette procédure. Le renouvellement et la continuité du Parlement sont garantis chaque année par la présence d'élèves de deux niveaux scolaires différents.

Le programme se développe en plusieurs phases, tout au long desquelles les élèves discutent des problèmes de leur village ou de leur ville. Parmi ceux-ci, ils sélectionnent les problèmes les plus importants et effectuent des propositions de solutions qui vont être exposées dans une session plénière dans la mairie devant le maire; de là surgit le défi, pour le maire, de travailler avec la collaboration des jeunes pour résoudre ces problèmes. Jusqu'à présent, les sujets qui ont le plus préoccupé les jeunes parlementaires ont été ceux en rapport avec les espaces de loisir, l'insécurité et le vandalisme, les drogues et l'environnement urbain. Par exemple, ils ont proposé de: «faire une discothèque pour enfants et adolescents », « construire une piscine municipale », « limiter la vitesse de circulation dans la ville» ou «faire des annonces contre les drogues dans lesquelles nous-mêmes soyons les protagonistes ».

$\mathrm{Vu}$ les résultats obtenus jusqu'à présent, on peut dire que le programme du Parlement des Jeunes fonctionne, en général, de façon satisfaisante, bien qu'il y ait des aspects qui doivent être approfondis dans de prochaines éditions, comme par exemple: stabiliser les parlements comme des structures permanentes de participation des jeunes; réaliser un travail plus direct, personnel et engagé pour satisfaire les propositions effectuées; intégrer de façon plus cohérente le projet dans le curriculum scolaire en essayant d'obtenir, à ce sujet, le soutien du professorat.

Le programme «Budgets Participatifs de Séville: Laboraforo ${ }^{14} »$ prend place dans le projet d'implantation des budgets participatifs municipaux de la ville de Séville, réalisé lors du changement de gouvernement municipal en 2003. Parallèlement au processus général de la mise en œuvre de cette initiative municipale $^{15}$ surgit l'idée de mettre en marche un processus qui implique les enfants

14. Le programme est géré par l'Équipe des Budgets Participatifs de Séville: «Laboraforo » (à consulter sur le site: http://www.grupo.us.es/laboraforo/).

15. On peut obtenir l'information sur l'initiative des Budgets Participatifs de la Mairie de Séville sur le site: http://www.presupuestosparticipativosdesevilla.org/. 
et les jeunes dans la gestion des budgets participatifs de la ville. Dans ce cas, par conséquent, le programme est adressé autant aux enfants qu'aux adolescents et jeunes.

Ce projet se structure en prenant comme référence l'école, mais il ne se limite pas exclusivement à celle-ci. De cette façon, les garçons et les filles qui font partie d'un autre type de collectifs d'enfants ou de jeunes (associations, clubs, etc.) peuvent s'y intégrer. Dans les différents établissements éducatifs ou collectifs de jeunes, on travaille à partir d'une série de matériaux didactiques avec le but de présenter cette expérience aux garçons et aux filles. Le travail des enfants et des jeunes aboutit à une série de propositions qui sont portées par leurs représentants aux assemblées générales des Budgets Participatifs de la ville. Les propositions sont généralement en rapport avec des aspects économiques et gestionnaires comme la dotation et l'amélioration des centres scolaires du quartier ou l'organisation d'ateliers et d'activités de divers types dans les centres civiques municipaux.

Il nous semble intéressant de souligner quelques aspects de ce programme qui le rendent singulier. D'abord, il n'y a pas de budget spécifique géré par les enfants et les jeunes mais ceux-ci font des propositions concernant le budget commun portées et défendues par eux-mêmes dans les assemblées des districts où les citoyens adultes débattent et votent. Cette procédure a la vertu de faire apparaître le processus plus réel à l'exception du fait que les enfants ne peuvent pas voter dans ces assemblées avant 16 ans. Ces expériences sont aussi particulièrement motivantes aussi bien grâce à l'esprit ludique qui les caractérise que grâce au caractère non formel des savoirs et des situations sur lesquels ils s'appuient qui s'avèrent plus intéressants que ceux habituellement rencontrés dans les salles de classe. Finalement, il est remarquable que toutes ces activités sont organisées de manière complètement ouverte, de sorte que les résultats qui en sont tirés constituent le fruit de l'initiative des garçons et des filles eux-mêmes et ne sont pas construits de façon uniquement réactive, c'est-à-dire, comme des réponses des enfants et des jeunes aux propositions des adultes.

\section{Questions posées}

Les expériences exposées révèlent, sans doute, l'intérêt éducatif des programmes qui visent à favoriser la participation des enfants, adolescents et jeunes dans leur propre espace urbain, et le rôle de ces programmes dans l'éducation des jeunes à la citoyenneté. Or, l'analyse de ces expériences permet de constater les difficultés existantes pour les lier plus étroitement à l'éducation scolaire, et promouvoir l'innovation du curriculum et des pratiques enseignantes. Les efforts d'innovation en rapport avec l'éducation à la citoyenneté rencontre en général de fortes résistances dues à différents facteurs, comme le manque d'une tradition curriculaire à ce sujet, les caractéristiques de la culture professionnelle des enseignants à ce propos et, en définitive, la « forme scolaire », qui se montre assez 
imperméable à l'incorporation d'initiatives éducatives favorisant l'intervention directe des élèves dans la réalité sociale. Dans ce contexte, nous pouvons avancer quelques premières conclusions issues de notre recherche ${ }^{16}$ en rapport avec les possibles difficultés et opportunités de l'enseignement et l'apprentissage de la participation des élèves dans les procédures de prise de décision aussi bien au sein de l'école qu'en dehors de celle-ci.

Avant tout, l'idée de participation n'est pas habituellement considérée comme un contenu important en rapport avec les savoirs scolaires qui doivent être appris par les élèves. Son incorporation aux activités scolaires peut être un peu forcée et déboucher sur un certain « jeu de simulation». Il est important de distinguer deux situations différentes: des expériences de participation dans la salle de classe et celles hors de la classe, dans l'environnement municipal, puisque les contextes et les formes de comportement dans les deux cas ont des caractéristiques différentes. Dans le cas de la classe, la dynamique scolaire traditionnelle n'admet pas facilement les innovations, et, si elle le fait, ces innovations risquent d'être dépourvues de leur signification. Elles sont faites, mais sans que leur réalisation affecte vraiment la logique du savoir scolaire dominant. Les expériences développées dans le contexte municipal sont aussi dominées par la dynamique politique habituelle, renvoyant à un comportement fréquent des citoyens comme « usagers» (non comme participants responsables) et un comportement fréquent des politiciens comme « dirigeants» qui accordent aux citoyens certains avantages mais sans assumer en profondeur le droit de ces citoyens à participer de façon responsable dans les affaires de leur ville.

La situation qui vient d'être décrite étant généralement la plus habituelle, nous la considérons comme un point de départ, dans une ligne de possible progression vers d'autres situations qui nous semblent plus désirables du point de vue éducatif. En ce sens, le rôle des formateurs impliqués nous semble très important pour dynamiser ce processus. Ils doivent avoir une vision claire de la situation et orienter les activités avec une vision stratégique conforme au projet éducatif, pour avancer dans une ligne de progression adéquate ${ }^{17}$.

D’autre part, puisque ces projets de participation constituent une tâche partagée et interactive, il faut tenir compte du fait que le processus formatif devrait affecter non seulement les enfants et les jeunes, mais aussi les politiciens (et les professeurs) impliqués. Le progrès, donc, vers une plus grande maturité des expériences de participation doit reposer sur des progrès respectifs des jeunes et des adultes,

16. Il s'agit de la recherche intitulée «Éducation à la citoyenneté et formation du professorat: Difficultés et possibilités d'éduquer dans la participation des citoyens », citée au début de cet article. Comme nous avons dit avant, nos conclusions procèdent, surtout, du projet du Parlement des Jeunes, et confirment, en tout cas, d'autres obtenues dans l'expérience des budgets participatifs. 17. En ce sens, l'idée de progression, de profonde tradition dans la didactique française, est très utile. Il est très intéressant, à cet égard, de consulter la proposition de Roger Hart (1997) concernant « l'escalier de participation », un modèle pour analyser la participation réelle des jeunes dans des projets dans lesquels ils sont insérés avec des adultes. 
de façon que les deux progrès soient mutuellement renforcés. En ce sens, il est important de penser la participation en termes de savoirs et compétences pour les intégrer dans le curriculum scolaire de façon explicite, ce qui n'est pas le cas actuellement Le contexte social, de sa part, ne fournit pas beaucoup d'occasions pour exercer ces habiletés, le modèle de démocratie en vigueur ne le facilitant pas et favorisant le comportement des citoyens comme usagers plutôt que comme participants engagés. La question donc de l'éducation à la participation soulève des questions éthiques, relatives aux savoirs scolaires et à l'identité professionnelle des enseignants.

Du point de vue éthique, les expériences de participation peuvent prendre une certaine tournure de «moralisation » des élèves c'est-à-dire de conformité aux normes sociomorales existantes, tendance qui semble être quelque peu inévitable de la part des enseignants. Or, nous avons pu constater que les enfants et les jeunes, une fois réellement plongés dans le « jeu » de la participation, ne sont pas facilement contrôlables par les éducateurs, ou par les politiciens, et développent des positions réellement critiques à l'égard des attitudes politiques existantes. Ces positions sont relativement ponctuelles à cause du caractère limité de ces expériences mais leur apparition soulève la question de leur exploitation pédagogique et des choix éthiques et politiques à faire de la part des enseignants.

En outre, les enseignants ont des difficultés pour intégrer, en profondeur, dans leurs pratiques pédagogiques des activités en rapport avec la participation. Les enseignants ont généralement une conception du savoir scolaire comme étant un ensemble fermé et complet, héritier d'une tradition qui légitime sa validité, et considèrent comme secondaires, de moindre valeur, des connaissances qui proviennent de la réalité sociale: ce qui est académique prévaut sur ce qui est quotidien, ce qui est théorique - ou spéculatif - sur ce qui est en rapport avec l'action. Les élèves, de leur part, quand ils jouent « le rôle d'élèves » dans le contexte scolaire savent que le savoir valide est le savoir académique, qui est habituellement utilisé dans ce contexte. Quand ils jouent « le rôle de citoyens », ils se confrontent à des situations quotidiennes bien connues, des situations liées à l'action, mais ils ne sont pas capables de transférer le savoir acquis à l'école pour le rendre utile et opérationnel dans ces situations. L'inverse est difficile aussi, à savoir utiliser l'expérience acquise à l'extérieure de la classe pour construire le savoir scolaire. De cette façon, le savoir scolaire apparaît comme étrange dans le contexte réel des citoyens et le savoir en rapport avec la participation réelle des citoyens apparaît comme étrange dans le contexte scolaire. Il y a donc une rupture mais aussi des tensions entre le contexte scolaire et le contexte social, qui rendent très difficile l'éducation des élèves à la participation.

Plus généralement, les enseignants se sentent peu sûrs dans des situations d'innovation qui ne leur sont pas suffisamment connues. Ils considèrent que leur fonction professionnelle est d'enseigner « le » savoir scolaire, avec lequel ils se sentent identifiés. Ainsi, par exemple, les activités qui nécessitent une certaine 
improvisation dans le cadre de l'éducation à la citoyenneté, comme les débats ou le traitement de questions problématiques (Oulton et al., 2004), semblent difficiles à gérer de la part des enseignants. Il s'agit d'activités qui impliquent un changement dans le rôle pédagogique de l'enseignant, ce qui affecte profondément sa propre «identité professionnelle ». Et cela n'est pas facile à assumer non plus. Il est pourtant important de constater l'incidence positive, sur le développement de l'enseignement, des expériences intéressantes de socialisation et de participation citoyennes vécues par les enseignants dans leurs propres contextes sociaux, non scolaires (Schugurensky and Myers, 2003).

L'éducation à la participation pose des problèmes complexes et ouvre plusieurs pistes de réflexion. Les tentatives pour changer le savoir scolaire doivent être accompagnées par des mesures qui visent aussi à la transformation du savoir professionnel des enseignants - comme cela est proposé dans le projet IRES (« Recherche et renouvellement scolaire ${ }^{18} »$ ), dont nous faisons partie. Construire un savoir scolaire désirable et construire un modèle d'enseignant désirable sont, donc, deux tâches étroitement liées.

Francisco F. García Pérez (Universidad de Sevilla. Facultad de Ciencias de la Educación) - Nicolás de Alba Fernández (Universidad de Sevilla. Facultad de Ciencias de la Educación) - Pilar Estada Aceña (Argos. Proyectos Educativos. Sevilla) - Trinidad Herrero Campos (Argos. Proyectos Educativos. Sevilla)

\section{BIBLIOGRAPHIE}

AUdigier François et LAGELÉE Guy. Éducation civique et initiation juridique dans les collèges. Paris: INRP (Didactique des disciplines), 1996.

Bolívar Antonio. Educación para la ciudadanía. Algo más que una asignatura. Barcelona: Graó (Crítica y Fundamentos), 2007.

DAm GeerT Ten and Volman Monique. «Critical Thinking as a Citizenship Competence: Teaching Strategies ». Learning and Instruction, volume XIV, Issue 4, August 2004, p. 359-379. <http://www.sciencedirect.com/science/journal/09594752> (page consultée le 16/11/2009).

DE AlBA Nicolás. « Educación para el Desarrollo, ¿una oportunidad para cambiar la educación? ». Investigación en la Escuela, nº 65, 2005, p. 63-71.

Delval Juan. Hacia una escuela ciudadana. Madrid: Morata (Pedagogía), 2006.

ÉDUSCOL. La citoyenneté par l'éducation. Actes du séminaire national (juillet 2006), Extraits 1/3. Paris: Ministère de l'Éducation nationale, de l'Enseignement supérieur et de la Recherche, 49 p. http://eduscol.education.fr

EuRYdicE. L'éducation à la citoyenneté à l'école en Europe. Bruxelles: Eurydice, 2005, 91 p. <http://www.educacionenvalores.org/IMG/pdf/CivicsFR.pdf> (page consultée le 16/11/2009).

18. Sur le Projet IRES - « Investigación y Renovación Escolar » - on peut consulter García, 2000. 
Ferreras Josechu, Herrero Trinidad, Estada Pilar, Montero Marcos y García Vanesa. « Parlamento Joven: Un espacio de participación juvenil en los Ayuntamientos ». Revista de Estudios de Juventud, nº 74, 2006, p. 185-202.

$<$ http://www.injuve.mtas.es/injuve/contenidos.downloadatt.action?id=1717338040> (page consultée le 16/11/2009).

GARCíA Francisco F. « Un modelo didáctico alternativo para transformar la educación: el Modelo de Investigación en la Escuela ». Scripta Nova, nº 64, 15 de mayo 2000, 19 p.

<http://www.ub.es/geocrit/sn-64.htm> (page consultée le 16/11/2009).

GARCía Francisco F. "El sentido de la educación como referente básico de la didáctica ». Investigación en la Escuela, no 55, 2005, p. 7-27.

HAHn Carole L. «What Can Be Done to Encourage Civic Engagement in Youth? ». Social Education, volume 65, Issue 2, mars 2001, p. 108-110.

<http://www.accessmylibrary.com/coms2/summary_0286-10967301_ITM> (page consultée le 16/11/2009).

HART Roger A. Children's Participation: the Theory and Practice of Involving Young Citizens in Community Development and Environmental Care. New York: Unicef/London: Earthscan, 1997.

JIMÉNEZ Juan R. et GONZÁLEZ Juan C. «Entre rhétorique et pratiques. L'éducation à la citoyenneté en Espagne ». Revue internationale d'éducation de Sèvres, nº 44 (L'élève, futur citoyen), avril 2007, p. 45-54.

Madrid: BOE. Ley Orgánica 2/2006, de 3 de mayo, de Educación. Boletin Oficial del Estado, nº 106, jueves 4 de mayo de 2006, p. 17158-17207.

MARTín Irene. Una propuesta para la enseñanza de la ciudadanía democrática en España. Madrid: Fundación Alternativas (Estudios de Progreso), 2006.

MoRin Edgard. Les sept savoirs nécessaires pour l'éducation du futur. Paris: Unesco, 1999.

NAVAl Concepción, PRINT Murray and IRIARTE Concepción. « Civic Education in Spain: A Critical Review of Policy ». Journal of Social Science Education, nº 2, 2003.

<http://www.jsse.org/2003-2/spain_naval.htm> (page consultée le 16/11/2009).

Oulton Christopher, Day Vanessa, Dillon Justin and GraCe Marcus. "Controversial Issues: Teachers'Attitudes and Practices in Context of Citizenship Education ». Oxford Review of Education, volume XXX, Issue 4, 2004.

$<$ http://www.informaworld.com/smpp/content?content=10.1080/0305498042000303973> (page consultée le 16/11/2009).

ROBERT François. Enseigner le droit à l'école. ESF, 1999 (Pédagogies en pratique).

SCHUGURENSKy Daniel and MYERS John. "Learning to Teach Citizenship: A Lifelong Learning Approach ». Encounters on Education, volume IV, Fall 2003, p. 145-166.

TORnEY-PURTA Judith and BARber Carolyn. " Democratic School Engagement and Civic Participation among European Adolescents: Analysis of Data from the IEA Civic Education Study ». Journal of Social Science Education, Special Edition: European Year of Citizenship through Education, 2005.

<http://www.jsse.org/2005-se/torney_purta_barber_iea_analysis.htm> (page consultée le 16/11/2009).

TonuCCI Francesco. La città dei bambini, Roma-Bari: Laterza, 1996. 